SCIPP-96-33

IEM-FT-138/96

hep-th/9608010

\title{
The problem of the stabilization of the dilaton in string theories *
}

\author{
J.A. $\operatorname{Casas}^{\dagger, \S}$ \\ $\S$ Santa Cruz Institute for Particle Physics \\ University of California, Santa Cruz, CA 95064, USA
}

\begin{abstract}
The crucial problem of how the dilaton field is stabilized at a phenomenologically acceptable value in string theories remains essentially unsolved. We show that the usual scenario of assuming that the dilaton is fixed by the (SUSY breaking) dynamics of just the dilaton itself (dilaton dominance scenario) is inconsistent unless the Kähler potential receives very important perturbative or non-perturbative contributions. Then, the usual predictions about soft breaking terms are lost, but still is possible to derive model-independent predictions for them.
\end{abstract}

SCIPP-96-33

IEM-FT-138/96

Jul 1996

${ }^{*}$ Talk given in the Fourth Conference on Supersymmetry (SUSY 96), Univ. of Maryland at College Park, May 29 - June 1, 1996. Research supported in part by: the CICYT, under contracts AEN950195; the European Union, under contract CHRX-CT92-0004.

${ }^{\dagger}$ On leave of absence from Instituto de Estructura de la Materia CSIC, Serrano 123, 28006 Madrid, Spain. 


\section{Introduction}

The crucial problem of how the dilaton field, $S$, whose expectation value gives the tree level gauge coupling constant at the string scale [1] ( $\left.\langle\operatorname{Re} S\rangle \sim g_{\text {string }}^{-2}\right)$ is stabilized at the experimentally "observed" value, $g_{\text {string }}^{-2} \simeq 2$, remains as a very challenging one. The dilaton potential is flat at all orders in perturbation theory as long as SUSY remains unbroken. On the other hand SUSY itself must be broken by non-perturbative effects, which strongly suggests that the same dynamics that breaks SUSY is also responsible for the stabilization of the dilaton (see ref.[2]).

In this sense a very economical and model-independent scenario is the so-called "Dilaton Dominance" [3, 4] one. This consists in assuming that SUSY is broken in the dilaton sector. In other words, only the $F_{S}$ auxiliary field is to take a non-vanishing VEV. Furthermore, the dilaton dependence of the Kähler potential, $K$, is assumed to be sufficiently well approximated by the tree-level expression, $K=-\log (S+\bar{S})$. These assumptions are completed with the phenomenologically mandatory one that the superpotential $W$ is in such an (unknown) way that the minimum of the potential, $V$, lies at an acceptable value for the dilaton円 $\left(\langle\operatorname{Re} S\rangle=g_{\text {string }}^{-2}\right)$ and at vanishing cosmological constant $(V=0)$. The previous assumptions lead to some interesting relationships among the different soft terms, with an automatical implementation of universality, something which is phenomenologically welcome for FCNC reasons [5].

The interest of this scenario raises two questions, which directly concern the problem of the stabilization of the dilaton:

i) Is there any form of the superpotential $W(S)$ (preferably with some theoretical justification) able to fulfill the previous requirements (i.e. a minimum of the scalar potential at $\langle\operatorname{Re} S\rangle \simeq 2$ and $V=0)$ ?

ii) How good is expected to be the tree-level approximation for $K(S, \bar{S})$ ?

Regarding the first question, $(i)$, I will survey in sect.2 a simple analytical argument (which has been presented in ref. [6]) that proves that, even if the potential has a minimum at $\langle\operatorname{Re} S\rangle \simeq 2$ and $V=0$, there must exist an additional minimum (or unbounded from below direction) in the perturbative region of $S$-values for which $V<0$. This can be proven for an arbitrary form $W(S)$. Similarly, we will show that the very existence of such a (local) minimum is forbidden unless a really huge conspiracy of different contributions to $W(S)$ takes place.

Concerning the second question, (ii), there are, unfortunately, indications that the stringy non-perturbative corrections to the Kähler potential may be sizeable [7, 8]. In principle, this may be good news since it could help to avoid the above-mentioned problems. The trouble here is that very little is known about the form of these nonperturbative corrections. The possibilities and predictions of such a scenario are explored in sections 3 and 4 .

\footnotetext{
${ }^{1}$ Here we are assuming that the Kac-Moody level of the gauge group is $k=1$, which is the most common possibility; otherwise $\langle\operatorname{Re} S\rangle=g_{\text {string }}^{-2} / k$.
} 


\section{Inconsistencies of the dilaton dominated scenario}

Taking the tree-level expression for the Kähler potential

$$
K=-\log (S+\bar{S})+\hat{K}\left(T, \bar{T}, \phi_{I}, \bar{\phi}_{I}\right),
$$

where $T, \phi_{I}$ denote generically all the moduli and matter fields respectively, the scalar potential in the dilaton-dominance assumption reads

$$
V=\frac{1}{2 \operatorname{Re} S}\left\{\left|(2 \operatorname{Re} S) W_{S}-W\right|^{2}-3|W|^{2}\right\}
$$

with $W_{S} \equiv \partial W / \partial S$. If the scenario is realistic, the previous potential should have a minimum at a realistic value of $S$, say $S_{0}$, with

$$
\operatorname{Re} S_{0}=\frac{1}{g^{2}} \simeq 2
$$

where, for simplicity of notation, $g$ denotes the gauge coupling constant at the string scale $\left(\sim 10^{17} \mathrm{GeV}\right)$. In addition, the vanishing of the cosmological constant, i.e. $V\left(S_{0}\right)=0$, implies

$$
\left|\left(2 \operatorname{Re} S_{0}\right) W_{S}\left(S_{0}\right)-W\left(S_{0}\right)\right|=\sqrt{3}\left|W\left(S_{0}\right)\right|
$$

and, thus

$$
\frac{2 \operatorname{Re} S_{0}}{\sqrt{3}+1} \leq\left|\frac{W}{W_{S}}\right|_{S=S_{0}} \leq \frac{2 \operatorname{Re} S_{0}}{\sqrt{3}-1}
$$

Performing the following change of variables

$$
z=e^{-\beta S} \quad(\beta \text { arbitrary })
$$

the physical region of $S$, i.e. $\operatorname{Re} S>0$, is mapped into the circle of radius 1 in the $z$-plane. The "realistic minimum" point, $z_{0}=e^{-\beta S_{0}}$, lies somewhere inside the circle. In the new variable, the functions $W, W_{S}$ are written as

$$
\begin{aligned}
W(S) & =\Omega(z) \\
W_{S}(S) & =\Omega_{S}(z) \equiv-\beta z \Omega^{\prime}(z)
\end{aligned}
$$

and condition (5) becomes

$$
\frac{2 \operatorname{Re} S_{0}}{\sqrt{3}+1} \leq\left|\frac{\Omega(z)}{\Omega_{S}(z)}\right|_{z=z_{0}} \leq \frac{2 \operatorname{Re} S_{0}}{\sqrt{3}-1}
$$

with $\operatorname{Re} S_{0}=-\log \left|z_{0}\right| / \beta$. Let us consider now the function

$$
\rho(z) \equiv \frac{\Omega(z)}{\Omega_{S}(z)} .
$$

Let us suppose for the moment that $\rho(z)$ is an analytical function with no poles inside the physical region $|z|<1$. Then, the maximum of $|\rho(z)|$ in the region $|z| \leq\left|z_{0}\right|$ must 
necessarily occur (principle of maximum) at some point $z_{M}$ belonging to the boundary, namely the circle $\mathcal{C} \equiv\left\{|z|=\left|z_{0}\right|\right\}$. If we consider the larger region enclosed by the broader circle $\mathcal{C}^{\prime} \equiv\left\{|z|=\left|z_{0}^{\prime}\right|\right\}$, with $\left|z_{0}^{\prime}\right|>\left|z_{0}\right|$, the new maximum of $|\rho(z)|$ must occur now at some point, say $z=z_{1}$, belonging to the boundary $\mathcal{C}^{\prime}$. From (8) it is clear that at $z_{1}$

$$
\left|\rho\left(z_{1}\right)\right|=\left|\frac{\Omega(z)}{\Omega_{S}(z)}\right|_{z=z_{1}}>\frac{2 \operatorname{Re} S_{0}}{\sqrt{3}+1}
$$

Taking the radius of $\mathcal{C}^{\prime}$ so that $\operatorname{Re} S_{1} \equiv-\log \left|z_{1}\right| / \beta=\frac{\sqrt{3}-1}{\sqrt{3}+1} \operatorname{Re} S_{0}$, we can write (10) as

$$
\left|\frac{\Omega(z)}{\Omega_{S}(z)}\right|_{z=z_{1}}=\left|\frac{W}{W_{S}}\right|_{S=S_{1}}>\frac{2 \operatorname{Re} S_{1}}{\sqrt{3}-1}
$$

Therefore, at $S=S_{1}$ the potential (2) has a negative value. On the other hand $S_{1}$ still belongs to the perturbative region

$$
\operatorname{Re} S_{1} \simeq 0.27 \operatorname{Re} S_{0}
$$

which means $\alpha \simeq 0.14$. The only way-out to the previous argument is to allow the function $\rho(z) \equiv \frac{\Omega(z)}{\Omega_{S}(z)}$ to have some pole in the region enclosed by $\mathcal{C}^{\prime}$. But then $\left|\frac{\Omega}{\Omega_{S}}\right| \rightarrow$ $\infty$ near the pole and necessarily $\left|\frac{\Omega}{\Omega_{S}}\right|>\frac{2 \operatorname{Re} S}{\sqrt{3}-1}$ at some point with non-zero $\Omega$. Hence we arrive to the same conclusion.

The previous argument shows that the realistic minimum assumed to take place in the usual dilaton-dominated scenario can never correspond to a global minimum. This is not certainly the most desirable situation.

We can go a bit further and show that under very general assumptions, the realistic point $S=S_{0}$ cannot correspond to $V\left(S_{0}\right)=0$.

From symmetry and analyticity arguments we know [7] that the non-perturbative superpotential must take the form $W=\sum_{i} d_{i} e^{-a_{i} S}$, so it is reasonable to assume that at the realistic point $\left(S_{0} \sim 2\right) W$ is dominated by one of the terms, say $W \sim e^{-a S}$ (as it happens for instance in usual gaugino condensation). Then, the vanishing of $\Lambda_{\cos }$ at $S=S_{0}$, eq.(四), implies

$$
\left(-a-\frac{1}{2 \operatorname{Re} S_{0}}\right)^{2}-\frac{3}{4\left(\operatorname{Re} S_{0}\right)^{2}}=0 .
$$

From (13) we obtain $a \simeq(\sqrt{3}-1) / 4$, absolutely incompatible with the requirement of a hierarchically small SUSY breaking (note that $\langle W\rangle \sim 1 \mathrm{TeV}$ requires $a \simeq 18$ ).

It could happen, however, that two o more terms of the form $W=\sum d_{i} e^{-a_{i} S}$ cooperate at the particular region $S \simeq 2$ to produce a more realistic scenariof. It is interesting to show that for this to happen in a dilaton dominated scenario a really

\footnotetext{
${ }^{2}$ This is the mechanism of the so-called racetrack models to generate SUSY breaking (see e.g. [2] and references therein). These models, however, lead naturally to moduli dominance SUSY breaking rather than dilaton dominance.
} 
huge conspiracy must take place. From eq.(5) we see that the condition $V\left(S_{0}\right)=0$ implies

$$
\left|W_{S}\right| \sim|W|
$$

Since $W_{S}=-\sum a_{i} d_{i} e^{-a_{i} S}$ and the condition of hierarchically SUSY breaking requires $a_{i} \gtrsim O(10)$, it is clear that a cancellation between terms with different exponents must occur inside $W_{S}$ for (14) to be fulfilled. Moreover, one has to demand that $S_{0}$ corresponds to a minimum, which implies $\partial V / \partial S=0$ and the determinant of the Hessian matrix $\mathcal{H}>0$. These two conditions imply in turn [6, 2]

$$
\begin{gathered}
(2 \operatorname{Re} S)^{2}\left|W_{S S}\right|=2|W| \\
\frac{2}{\sqrt{3}}(\operatorname{Re} S)^{2}\left|W_{S S S}\right| \sim\left|W_{S}\right| \sim|W| .
\end{gathered}
$$

These requirements are completely unnatural since the typical sizes of $W_{S S}$ and $W_{S S S}$ are $a_{i}^{2} W, a_{i}^{3} W$ respectively, i.e. much larger that $W$. Therefore, two unpleasant finetunings must occur at $S_{0}$ to become a (local) minimum of the potential (for related work see ref. $[9])$.

To summarize the results of this section, the standard (tree-level) dilaton-dominated scenario can never correspond to a global minimum of the potential at $V=0$. Similarly, under very general assumptions it cannot correspond to a local minimum either, unless a really big conspiracy of different contributions to $W(S)$ takes place.

\section{The role of the Kähler potential and a predictive scenario}

The previous results, plus the fact that the Kähler potential is likely to receive sizeable string non-perturbative corrections, strongly suggest to consider a more general scenario, as commented in the introduction. Thus, in this section we will study how far we can go with the usual assumption of "dilaton-dominance" (i.e. only $\left|F_{S}\right| \neq 0$ ), but leaving the Kähler potential arbitrary. The potential reads

$$
V=K_{S \bar{S}}\left|F_{S}\right|^{2}-3 e^{K}|W|^{2}
$$

where $F_{S}=e^{K / 2}\left\{\left(K^{-1}\right)^{\bar{\phi} S}\left(\partial_{\phi} W+W K_{\phi}\right)^{*}\right\}$ with $\phi$ running over all the chiral fields and the subindices denoting partial derivatives. If we also assume vanishing cosmological constant, then

$$
\left|F_{S}\right|\left(K_{S \bar{S}}\right)^{1 / 2}=\sqrt{3} e^{K / 2}|W|=\sqrt{3} m_{3 / 2} .
$$

The effective low-energy soft part of the Lagrangian (in terms of the canonically normalized fields) is given by

$$
\begin{aligned}
-\quad \mathcal{L}_{\text {soft }} & =\frac{1}{2} M_{1 / 2}^{a} \hat{\hat{\lambda}}_{a} \hat{\lambda}_{a}+\sum_{I} m_{I}^{2}\left|\hat{\phi}_{I}\right|^{2} \\
+\quad & \left(A_{I J L} Y_{I J L} \hat{\phi}_{I} \hat{\phi}_{J} \hat{\phi}_{L}+\text { h.c. }\right)+\cdots
\end{aligned}
$$


The values of the gaugino masses, $M_{1 / 2}^{a}$, scalar masses, $m_{I}^{2}$, and coefficients of the trilinear scalar terms, $A_{I J L}$, can be computed using general formulae [3, 10, and eqs. 1718) (for more details see ref. [6])

$$
\begin{aligned}
m_{I}^{2} & =m_{3 / 2}^{2} \\
\left|\frac{M_{1 / 2}}{m_{3 / 2}}\right| & =\left[\frac{3 g^{4}}{4 K_{S \bar{S}}}\right]^{1 / 2} \\
\left|\frac{M_{1 / 2}}{A}\right| & =\left|\frac{g^{2}}{2 K_{S}}\right| .
\end{aligned}
$$

Here we have assumed that the Yukawa couplings appearing in the original superpotential, $W$, do not depend on the dilaton $S$. This is true at tree-level (and thus at the perturbative level) and, since they are parameters of the superpotential, they are not likely to be appreciably changed at the non-perturbative level [7]. The expression for the coefficient of the bilinear term, $B$, depends on the mechanism of generation of the $\mu$ term, so we prefer to leave it as an independent parameter. Finally, in order to fulfill the phenomenological requirement of (approximate) universality [5] we have demanded that $K_{I \bar{I}}$ does not get $S$-dependent contributions?. Notice also that in the tree level limit $K_{S}=\frac{1}{2 \operatorname{Re} S}, K_{S \bar{S}}=\frac{1}{4(\operatorname{Re} S)^{2}}$, and we recover from eqs.(20) the usual tree level relations [3, 田.

In eqs.(20) there are three unknowns $\left(m_{3 / 2}, K_{S}, K_{S \bar{S}}\right)$ and three soft breaking parameters $\left(m_{I}^{2}, M_{1 / 2}, A\right)$. On the other hand, the non-perturbative superpotential must take the form $W=\sum_{i} d_{i} e^{-a_{i} S}$, so, as explained above, it is reasonable to assume that at the realistic point, $S \sim 2, W$ is dominated by one of the terms, say $W \sim e^{-a S}$, as it happens for instance in usual gaugino condensation. Thus, the condition $\Lambda_{\text {cos }}=0$, i.e. eq.(18), gives us a further constraint, namely

$$
\left|-a+K_{S}\right|^{2}-3 K_{S \bar{S}}=0
$$

which relates the values of $K_{S}, K_{S \bar{S}}$ and $a$. Notice that the latter is essentially fixed by the condition of a hierarchical SUSY breaking $(a \simeq 18)$, thus eqs.(20) and eq.(21) give a non-trivial scenario whose phenomenology could be investigated.

\section{Ansatzs for non-perturbative Kähler potentials}

It is tempting to go a bit further in our analysis and explore the phenomenological capabilities of explicit (stringy) non-perturbative effects on $K$ which have been suggested in the literatureft.

From the arguments explained in ref.[7], it is enough to focuss our attention on possible forms of $K(S+\bar{S})$, exploring the chances of getting a global minimum at $V=0$.

\footnotetext{
3 This, of course, may not occur. However, it is a common assumption of all existing stringbased models (including the usual dilaton-dominance model). It should be noted however that the universality of the scalar masses (unless that of gaugino masses) is something imposed, rather than obtained from the model.

${ }^{4}$ For other attempts in this sense, see ref. 11.
} 
From eq.(21) we can check that a successful generalized dilaton dominated scenario (i.e. with $V=0$ at $\operatorname{Re} S \simeq 2$ ) requires very sizeable non-perturbative corrections to $K$. This can be seen by considering the form of eq.(21) when $K$ is substituted by the tree level expression $K=-\log (S+\bar{S})$. The fact that $a \simeq 18$ implies that the non-perturbative "corrections" to $K_{S}, K_{\bar{S} S}$ must indeed be bigger than the tree level values. If we further demand that the potential has a minimum at $V=0$ this implies

$$
\begin{aligned}
\left(-a+\frac{1}{2} K^{\prime}\right) K^{\prime \prime}-\frac{3}{4} K^{\prime \prime \prime} & =0 \\
\left(-a+\frac{1}{2} K^{\prime}\right)^{2}-\frac{3}{4} K^{\prime \prime} & =0
\end{aligned}
$$

(at the realistic point $\operatorname{Re} S=2$ ), where the primes denote derivatives with respect to $\operatorname{Re} S$ (the second equation is simply eq.(21)). It would be nice if the previous conditions could be fulfilled by some simple form of $K$.

The fact that the SUGRA lagrangian has an exponential dependence on $K$ (e.g. it can be written as $\mathcal{L}=\left[e^{-K / 3}\right]_{D}$ ) together with the fact that in the known examples (mainly orbifold compactifications) the perturbative corrections to $K$ are small, suggest that a sensible decomposition for $K$ is

$$
e^{K}=e^{K_{t r e e}}+e^{K_{n p}}
$$

where the first term corresponds to the tree level expression $\left(K_{\text {tree }}=-\log (2 \operatorname{Re} S)\right)$ and $K_{n p}$ denotes the non-perturbative contributions. The next step is to choose some plausible form for $K_{n p}(\operatorname{Re} S)$ and to study the form of $V$, see eq.(17). The field theory contributions to $K_{n p}$ have been evaluated in ref. [12], but for a realistic case they turn out to be too tiny to appreciably modify the tree-level results" according to the work of ref. [8], stringy non-perturbative effects may be sizeable (even for weak four-dimensional gauge coupling) and plausibly go as $g^{-p} e^{-b / g}$ with $p, b \sim$ $O(1)$. Then a simple possibility is to take

$$
e^{K_{n p}}=d g^{-p} e^{-b / g}
$$

where $d, p, b$ are constants with $p, b>0$ and $g^{-2}=\operatorname{Re} S$.

The numerical results indicate that, plugging this ansatz, it is not difficult to get a minimum of the potential using just one condensate with $a \simeq 18$ (thus guaranteeing the correct size of SUSY breaking) and sensible values for the $d, p, b$ constants. More precisely, for $d=-3, p=0, b=1$, there is a minimum of the potential at $\operatorname{Re} S=2.1$. Unfortunately, the negative value of $d$ makes this example unacceptable. Another example with positive $d$ is $d=7.8, p=1, b=1$, which has a minimum at $\operatorname{Re} S=1.8$. However, as a general result, playing just with these simple forms for $K$, it seems impossible to get the minimum at $V=0$. Anyway, it is impressive that just with

\footnotetext{
${ }^{5}$ More precisely, the authors of ref. [12] obtain $K=-3 \log \left[(2 \operatorname{Re} S)^{1 / 3}+e^{-K_{p} / 3}+d e^{-(2 \operatorname{Re} S) / 2 c}\right]$,
} where $K_{p}$ denotes the perturbative corrections to the tree-level expression, $d$ is an unknown constant and $c$ is essentially the coefficient of the one-loop beta function (the non-perturbative superpotential goes as $\left.e^{-3 S / 2 c}\right)$. 
one condensate and choosing very reasonable values for $d, p$ and $b$ (note also that there is no fine-tuning in the previous choices), a minimum appears at the right value of the dilaton. Another typical characteristic of these examples is the appearance of singularities in the potential caused by zeroes of the second derivative of the Kähler potential, $K^{\prime \prime}$, at some particular values of $S$. Of course, this can be cured by additional terms in $K$.

Finally, it would be worth to analyze the possible implications of this kind of nonperturbative Kähler potentials for other different matters, such as the cosmological moduli problem [13, 14.

\section{References}

[1] E. Witten, Phys. Lett. B155 (1985) 151.

[2] B. de Carlos, J.A. Casas and C. Muñoz, Nucl. Phys. B399 (1993) 623.

[3] V. Kaplunovsky and J. Louis, Phys. Lett. B306 (1993) 269.

[4] A. Brignole, L.E. Ibáñez and C. Muñoz, Nucl. Phys. B422 (1994) 125 [Erratum: B436 (1995) 747].

[5] S. Dimopoulos and H. Georgi, Nucl. Phys. B193 (1981) 150; F. Gabbiani, E. Gabrielli, A. Masiero and L. Silvestrini, hep-ph/9604387; J.A. Casas and S. Dimopoulos, hep-ph/9606237, to appear in Physics Letters B.

[6] J.A. Casas, hep-th/9605180, to appear in Physics Letters B.

[7] T. Banks and M. Dine, Phys. Rev. D50 (1994) 7454.

[8] S.H. Shenker, Proceedings of the Cargese Workshop on Random Surfaces, Quantum Gravity and Strings, Cargese (France) 1990.

[9] R. Brustein and P.J. Steinhardt, Phys. Lett. B302 (1993) 196; A. de la Macorra, hep-ph/9501250.

[10] L. Hall, J. Lykken and S. Weinberg, Phys. Rev. D27 (1983) 2359; S.K. Soni and H.A. Weldon, Phys. Lett. B126 (1983) 215.

[11] Z. Lalak, A. Niemeyer and H.P. Nilles, Phys. Lett. B349 (1995) 99; Nucl. Phys. B453 (1995) 100; P. Binetruy, M.K. Gaillard and Y. Wu, hepth/9605170.

[12] C.P. Burgess, J.P. Derendinger, F. Quevedo and M. Quirós, hep-th/9505171.

[13] T. Banks, D.B. Kaplan and A.E. Nelson, Phys. Rev. D49 (1994) 779.

[14] B. de Carlos, J.A. Casas, F. Quevedo and E. Roulet, Phys. Lett. B318 (1993) 447. 